\title{
Relaxation Patterns of Nearly Critical Gels
}

\section{Marian Mours and H. Henning Winter*}

Department of Chemical Engineering, University of Massachusetts at Amherst, Amherst, Massachusetts 01003

Received November 16, 1995; Revised Manuscript Received August 5, $1996^{\otimes}$

\begin{abstract}
We measure the complex rheological behavior of nearly critical gels and analyze the data by searching for characteristic patterns and abstracting those patterns into a self-consistent model. The sample is a linear, flexible, nearly monodisperse polybutadiene which gets cross-linked on its vinyl side groups. The dynamic mechanical storage and loss moduli of cross-linking polymers change smoothly during the liquid-solid transition, while equilibrium rheol ogical properties (e.g., zero-shear viscosity and equilibrium compliance) diverge. During gelation, the relaxation occurs in a distinct pattern which can be described in a quantitative way with a minimum number of parameters. The pattern can be understood as a combination of the BSW spectrum (representing the precursor relaxation behavior) and the selfsimilar Chambon-Winter gel spectrum (modeling the terminal relaxation due to growing clusters). The spectrum is cut off at the material's longest relaxation time, $\lambda_{\max }$. Our model parameters, $\lambda_{\max }$ and $\mathrm{G}_{\mathrm{e}}$ (equilibrium modulus), exhibit characteristic scaling behavior with respect to the distance from the gel point, $\left|p-p_{c}\right|$. The relaxation exponent, $n$, in the terminal zone is a function of the extent of reaction. Hence, dynamic scaling (requires constant $n$ values) is not valid for our system. The proposed model passes the self-consistency test by predicting the mechanical behavior (at different frequencies) as a function of the extent of reaction and other rheological observations during the sol-gel transition.
\end{abstract}

\section{Introduction}

A cross-linking material is able to form molecular clusters which grow in size. A transition from a liquid to a solid state occurs when the largest cluster diverges in size at a sufficiently high degree of cross-linking, $\mathrm{p}_{\mathrm{c}}$. The material at this transition (gel point, GP) is known as a critical gel. Before the gel point, the liquid-like behavior can be described by the zero-shear viscosity, $\eta_{0}$, which increases during cross-linking until it diverges at the gel point. Beyond the gel point, the now solid material is characterized by the growing equilibrium modulus, $G_{e}$, which is zero before and at the GP. Critical gels exhibit an unusually simple and regular relaxation behavior which expresses itself in a selfsimilar relaxation modulus that was experimentally observed by Chambon and Winter, ${ }^{1-3}$

$$
\mathrm{G}(\mathrm{t})=\mathrm{St}^{-\mathrm{n}}
$$

where $\mathrm{S}$ is commonly called the gel stiffness and $\mathrm{n}$ is the critical relaxation exponent. The self-similar relaxation behavior is valid in the terminal zone at long times, $\lambda_{0}<\mathrm{t}<\infty$. The lower limit corresponds to some relaxation time that characterizes the crossover to a different relaxation mechanism. In the case of low molecular weight precursors $\left(\mathrm{M}_{\mathrm{w}}<\mathrm{Me}_{\mathrm{e}}\right)$, this is the crossover to the glass transition; for higher molecular weight precursors $\left(M_{w}>M_{e}\right), \lambda_{0}$ characterizes the crossover to entanglement relaxation. ${ }^{4}$ Since the longest relaxation time diverges at the gel point, the selfsimilar relaxation is valid to infinite time. The selfsimilar relaxation behavior can also be expressed by the power law relaxation time spectrum (CW spectrum): ${ }^{1-3}$

$$
\mathrm{H}(\lambda)=\frac{\mathrm{S}}{\Gamma(\mathrm{n})} \lambda^{-\mathrm{n}} \quad \lambda_{0}<\lambda<\infty
$$

The relaxation exponent may adopt values $0<\mathrm{n}<1$. Relaxation exponents less than zero would lead to an unphysical increase in the relaxation modulus with

\footnotetext{
${ }^{\otimes}$ Abstract published in AdvanceACS Abstracts, September 15, 1996.
}

time, and the upper limit of $n<1$ guarantees the divergence of the viscosity at the gel point. Consequently, the dynamic storage and loss moduli, $\mathrm{G}^{\prime}$ and $\mathrm{G}^{\prime \prime}$, scale with frequency, $\omega$, at the gel point:

$$
\mathrm{G}^{\prime}=\frac{\mathrm{G}^{\prime \prime}}{\tan \delta}=\mathrm{S} \Gamma(1-\mathrm{n}) \cos \frac{\mathrm{n} \pi}{2} \omega^{\mathrm{n}} \quad 1 / \lambda_{0}>\omega>0
$$

This leads to a loss tangent which is independent of frequency at the gel point and directly related to the relaxation exponent $n$ :

$$
\tan \delta=\tan \mathrm{n} \pi / 2
$$

Due to the critical nature of the liquid-solid transition, transient rheological properties such as zero-shear viscosity and equilibrium modulus show a distinct scaling behavior in the vicinity of the gel point:5,6

$$
\begin{array}{ll}
\eta_{0} \propto\left(p_{c}-p\right)^{-s} & p<p_{c} \\
G_{e} \propto\left(p-p_{c}\right)^{z} & p>p_{c}
\end{array}
$$

Dynamic scaling based on percolation theory ${ }^{7-9}$ predicts different values for $s$ and $z$, depending on the specific model assumption. Martin and Adolf $\mathrm{f}^{10}$ report $\mathrm{n}=2 / 3, \mathrm{~s}$ $=4 / 3$, and $z=8 / 3$ in the case of Rouse behavior (complete screening of hydrodynamic interactions), $n=1, s=0$, and $z=8 / 3$ for Zimm behavior (hydrodynamic interactions included), and $n=0.71, s=0.75$, and $z=1.94$ if the electrical network analogy introduced by de Gennes ${ }^{11}$ is used. Classical mean-fiel d gelation theory $\mathrm{y}^{12,13}$ based on the Bethe lattice predicts ${ }^{10} \mathrm{n}=1, \mathrm{~s}=0$, and $\mathrm{z}=3$. De Gennes ${ }^{14}$ predicted that percolation theory should hold for cross-linking of small-molecule precursors. However, he argued that, for vulcanizing polymers (high $M_{w}$ ), only a very narrow regime near the gel point exists for which percolation is valid, i.e., these polymers should exhibit more mean-field-like behavior.

Winter ${ }^{15}$ used the scaling of $\eta_{0}$ and $\mathrm{G}_{\mathrm{e}}$ to describe the behavior of the longest relaxation time, $\lambda_{\max }$, in the vicinity of the gel point. He derived from experimental 
observations that $\lambda_{\max }$ scal es with the extent of reaction as

$$
\begin{array}{ll}
\lambda_{\max } \propto\left(p_{c}-p\right)^{-\alpha_{-}}, \text {with } \alpha_{-}=s /(1-n) & p<p_{c} \\
\lambda_{\max } \propto\left(p-p_{c}\right)^{-\alpha_{+}}, \quad \text { with } \alpha_{+}=z / n & p>p_{c}
\end{array}
$$

This may be simplified by postulating symmetrical scaling before and after the gel point, $\alpha_{-}=\alpha_{+}$, which results in a relation between the different gel exponents: 15

$$
\mathrm{n}=\mathrm{z} /(\mathrm{s}+\mathrm{z})
$$

Since the longest relaxation time is difficult to assess experimentally, this assumption has never been proven directly. Durand et al. ${ }^{16}$ and Gol denfeld and Goldbart ${ }^{17}$ derived the same relation in different ways. One could invert the above arguments and say that, if eq 9 is true, the longest relaxation time diverges symmetrically on both sides of the gel point. Experimentally, it has been shown that eq 9 holds for cross-linking of very low molecular weight precursors. ${ }^{18}$

Several models for the relaxation behavior of nearly critical and critical gels have been presented. ${ }^{4,19-24}$ None of these models was able to describe all experimental observations in the vicinity of the gel point. Either the models were restricted to the material at the gel point, or they were developed for small-molecule precursors only, or they simply did not yield good agreement with experimental data.

In this study, we explore the complex rheological behavior of nearly critical gels from high molecular weight precursors by observing relaxation patterns and subsequently formulating a phenomenological model with a small number of meaningful parameters. This search for patterns results in a unified picture, i.e., the complex rheological behavior gets abstracted into a simple model which governs all rheological observations near the gel point. Mapping of the model properties and self-consistency are important aspects in the development and application of the new model. The new model can subsequently be used to study the origins of the observed behavior using molecular approaches such as molecular dynamics simulations. The unified and comprehensive picture presented by such a model simplifies comparison of experiment and theory.

\section{Experimental Section}

Linear, flexible, nearly monodisperse polybutadienes with two different molecular weights (PBD18, $\mathrm{M}_{\mathrm{w}}=18000$, and PBD38, $M_{w}=38000$ ) were cross-linked with a bifunctional cross-linker, p-bis(dimethylsilyl)benzene at the pendant 1,2vinyl units that are randomly placed along the backbone of the chain. The characterization of the polybutadiene precursors is given elsewhere. ${ }^{4}$ The cross-linking reaction was catalyzed by a platinum compound, cis-dichlorobis(diethylsulfide)platinum(II), which was applied as an $8 \times 10^{-6} \mathrm{M}$ solution in toluene. Samples were prepared by mixing $0.6 \mathrm{~g}$ of polymer precursor with $0.03 \mathrm{~mL}$ of catalyst solution in a ceramic crucible using a microspatula. The solvent was removed from the mixture by vacuum stripping at $70^{\circ} \mathrm{C}$ for $2 \mathrm{~h}$. The mixture was then allowed to cool to room temperature before the stoichiometric amount of cross-linker was added. In this study, a stoichiometric ratio (ratio of the number of functional groups of the cross-linker to the number of vinyl groups per polymer chain) of unity was chosen for all experiments. The addition of the cross-linker started the reaction. The mixture was stirred for 6 min before it was used in an experiment.
Rheological studies were performed both during the reaction and after having stopped the reaction at intermediate conversions. Reacting samples were investigated at $28^{\circ} \mathrm{C}$ using a time-resolved rheometry technique ${ }^{25}$ in a Rheometrics dynamical spectrometer, RDS 7700, equipped with parallel plates (diameter $25 \mathrm{~mm}$ ). Cyclic frequency sweeps (CF Ss) probed the samples in a frequency window of 1-500 rad/s. Stopped samples were prepared as follows: The reacting mixture was poured into a mold with a diameter of $25 \mathrm{~mm}$ and a depth of about $0.8 \mathrm{~mm}$. This mold consists of a bottom rheometer plate and an aluminum ring which was machined to fit tightly around the plate. The gap between plate and ring was sealed with a peroxide-cured rubber O-ring. At the desired reaction time, an 80 wt \% solution of tetramethylethylenediamine (TMEDA), a catalyst poison, in toluene was sprayed onto the sample. The poison rapidly diffused into the sample and thereby effectively stopped the cross-linking reaction. The average diffusion time of $1-2 \mathrm{~min}$ is considerably smaller than the overall reaction time to reach the gel point $(\sim 1 \mathrm{~h})$. The aluminum ring was then removed, and the sample was placed into the rheometer. The dynamic mechanical response of a variety of stopped samples of both PBD18 and PBD38, was measured at temperatures between -75 and $50{ }^{\circ} \mathrm{C}$.

The chemical extent of reaction during cross-linking was monitored by FTIR spectroscopy. The reacting mixture was placed between two $\mathrm{KBr}$ plates in an IBM IR/32 infrared spectrometer at $28{ }^{\circ} \mathrm{C}$ which took a spectrum every 3-5 min at a resolution of $4 \mathrm{~cm}^{-1}$. The reduction in absorbance at around $2120 \mathrm{~cm}^{-1}$ is linearly proportional to the depletion of the cross-linker's silane group. This is a measure of the extent of reaction. ${ }^{26}$ The validity of Beer's law (linearity between absorbance and concentration) was tested with a set of welldefined samples of different cross-linker concentrations. The extent of reaction is calculated from the height of the silane absorption band at time $t$ and the initial height at time $t=0$ (taken from the calibration curve). A direct correlation between rheological data and extent of reaction was possible by simultaneously performing rheological and infrared experiments on the same sample. The temperatures of rheometer and spectrometer were calibrated prior to the experiments to agree within $\pm 0.5 \mathrm{~K}$.

All rheological data were analyzed using IRIS ${ }^{27}$ and GEL$\mathrm{PRO}^{25}$ software. Stopped sample data at different temperatures shifted onto master curves, and relaxation time spectra were subsequently calculated using the parsimonious model.28 CFS experiments on cross-linking samples were analyzed using GELPRO. The CFS data are split into separate sets (one for each experimental frequency) to follow the evolution with time at different frequencies. Frequency-dependent data can then be interpolated at any given time of the experiment, i.e., at any stage of cross-linking, resulting in an instantaneous rheological picture of the material.

\section{Experimental Results}

I nfrared spectra during the cross-linking reaction of PBD38, Figure 1, show the depletion of cross-linker silane groups reflected in the decrease of the absorption band at $2120 \mathrm{~cm}^{-1}$. The reaction of vinyl units causes the decrease of the absorption band at $1637 \mathrm{~cm}^{-1}$. The fractional extent of reaction was calculated from the height at time $t$ and the initial height at time $t=0$ (from calibration curve) of the $2120 \mathrm{~cm}^{-1}$ absorption band, since this decrease is more pronounced than that of the vinyl absorption band at $1637 \mathrm{~cm}^{-1}$ :

$$
p(t)=\frac{h\left(t_{0}\right)-h(t)}{h\left(t_{0}\right)}
$$

Figure 2 shows the resulting dependence of the fractional extent of reaction, $p$, on reaction time, $t$, for the case of PBD38. 


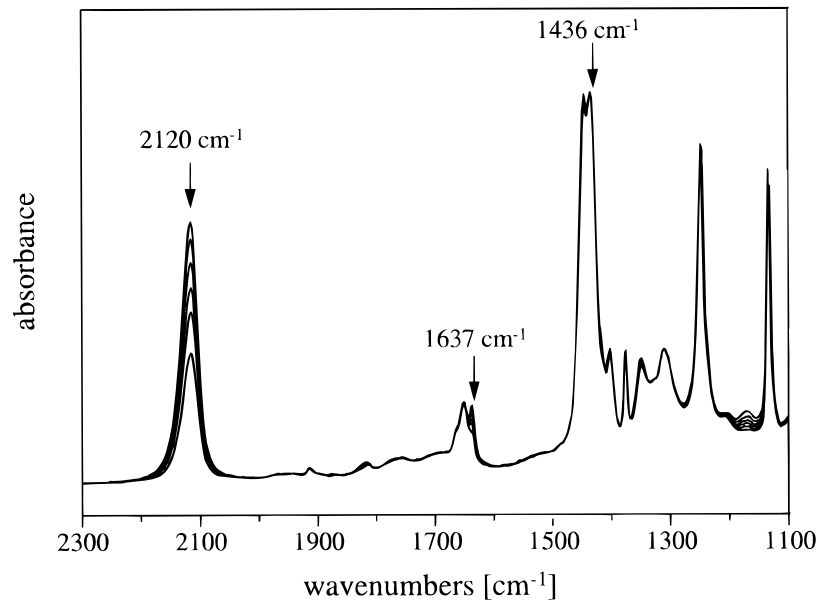

Figure 1. Infrared absorption spectra of cross-linking PBD38. The absorbance band at $2120 \mathrm{~cm}^{-1}$ corresponds to the silane groups of the cross-linker, and the $1637 \mathrm{~cm}^{-1}$ peak results from $\mathrm{C}=\mathrm{C}$ stretch of the pendant vinyl units on the polymer.

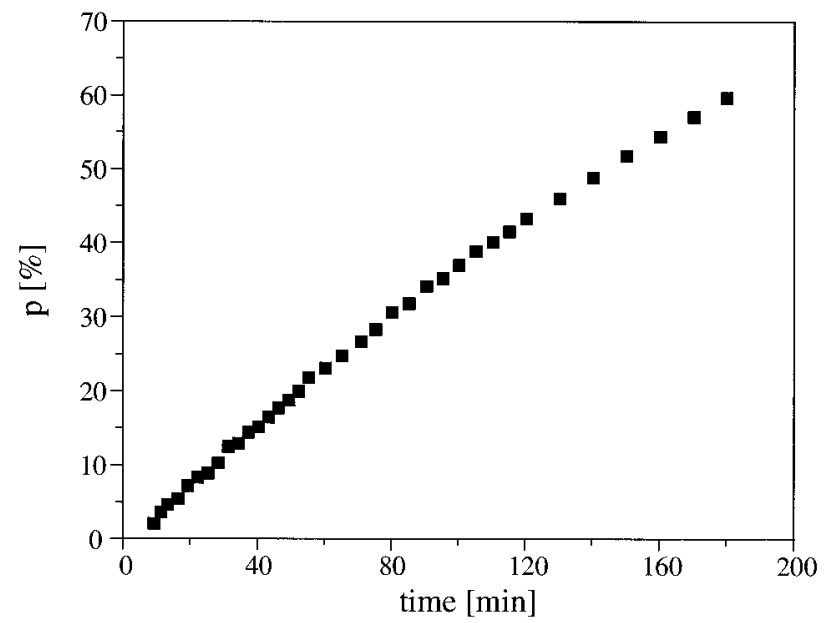

Figure 2. Fractional extent of reaction vs time as determined from FTIR spectra (see eq 10) for cross-linking PBD38.

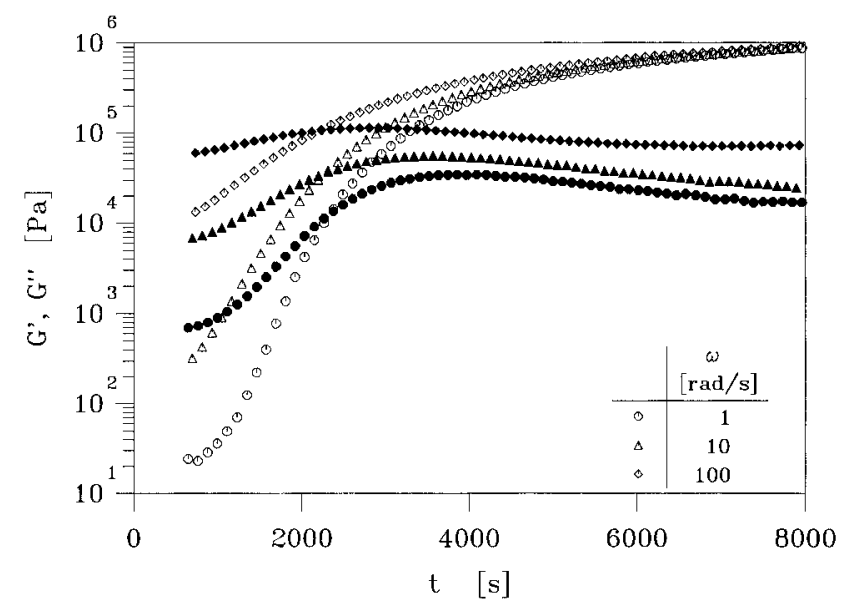

Figure 3. E volution of dynamic moduli during cross-linking of PBD38 at $28^{\circ} \mathrm{C}$. Data at three different frequencies are shown (open symbols, G'; filled symbols, $\mathrm{G}^{\prime \prime}$ ).

Dynamic storage and loss moduli, $\mathrm{G}^{\prime}$ and $\mathrm{G}^{\prime \prime}$, typically evolve as shown in Figure 3 for PBD38 at three different frequencies. A corresponding data set exists for PBD18. The gel time was taken from the crossover of $\tan \delta$ curves at the lower frequencies, as described above (see eq 4). This results in gel times of $53 \mathrm{~min}$ for PBD18 and $41 \mathrm{~min}$ for PBD38 which correspond to critical extents of reaction, $p_{c}$, of $20.3 \%$ for PBD18 and $15.7 \%$
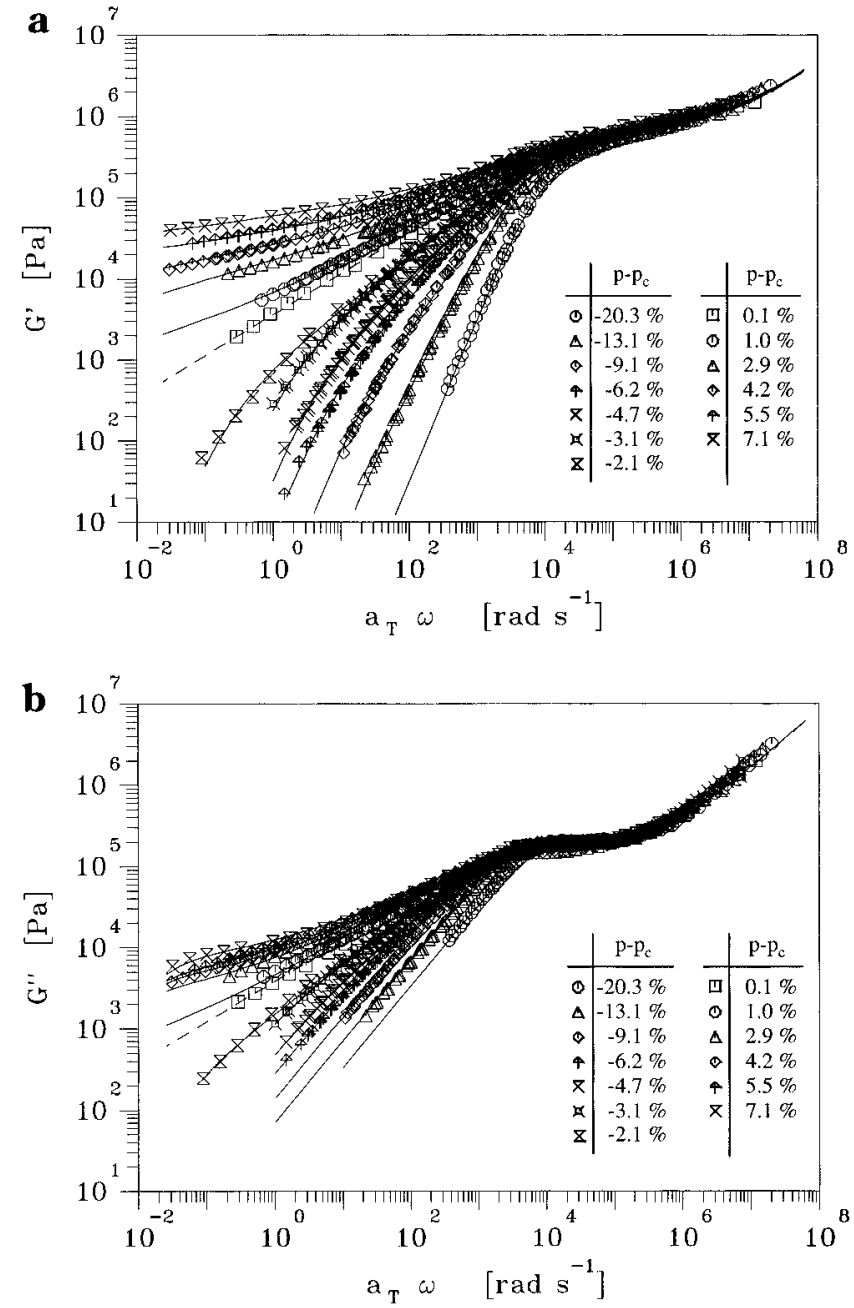

Figure 4. Dynamic moduli data (symbols) and model (line) of stopped PBD18 samples at different extents of reaction ( $T_{\text {ref }}$ $=28^{\circ} \mathrm{C}$ ): (a) storage modulus, $\mathrm{G}^{\prime}$; (b) loss modulus, $\mathrm{G}^{\prime \prime}$. The gel point is marked by a dashed line.

for PBD38. The relaxation exponent was calculated from the value of $\tan \delta$ at the gel point (eq 4) (PBD18, $\mathrm{n}=0.5$; PBD38, $\mathrm{n}=0.45$ ).

Master curves for stopped samples of PBD18 and PBD38, Figures 4 and 5, respectively, could be constructed using time-temperature superposition. A dashed line marks the gel point. The shift factors are very similar for samples at different stages of crosslinking (Figure 6). This completely agrees with previous results on linear and branched polybutadienes. ${ }^{29,30}$ It is evident that the most important changes occur in the terminal zone, while the entanglement relaxation behavior is only marginally affected. This is probably due to the small number of chemical cross-links added compared to the number of entanglements (temporary cross-links) already present in the precursor material. ${ }^{4}$

Using GELPRO software, ${ }^{25}$ frequency-dependent dynamic moduli from the timeresolved rheometry experiment could be interpolated at discrete times, $\mathrm{t}_{\mathrm{i}}$. Each interpolated data set can be viewed as a "snapshot" of the material at a certain intermediate reaction stage. This allows comparison (within the CFS frequency window) with dynamic moduli master curves of a stopped sample at the same extent of reaction. Figure 7 shows this superposition of stopped sample master curves and interpolated dynamic moduli for several PBD38 samples. Equivalent reaction times $t_{i}$ were determined for the stopped samples by matching them 

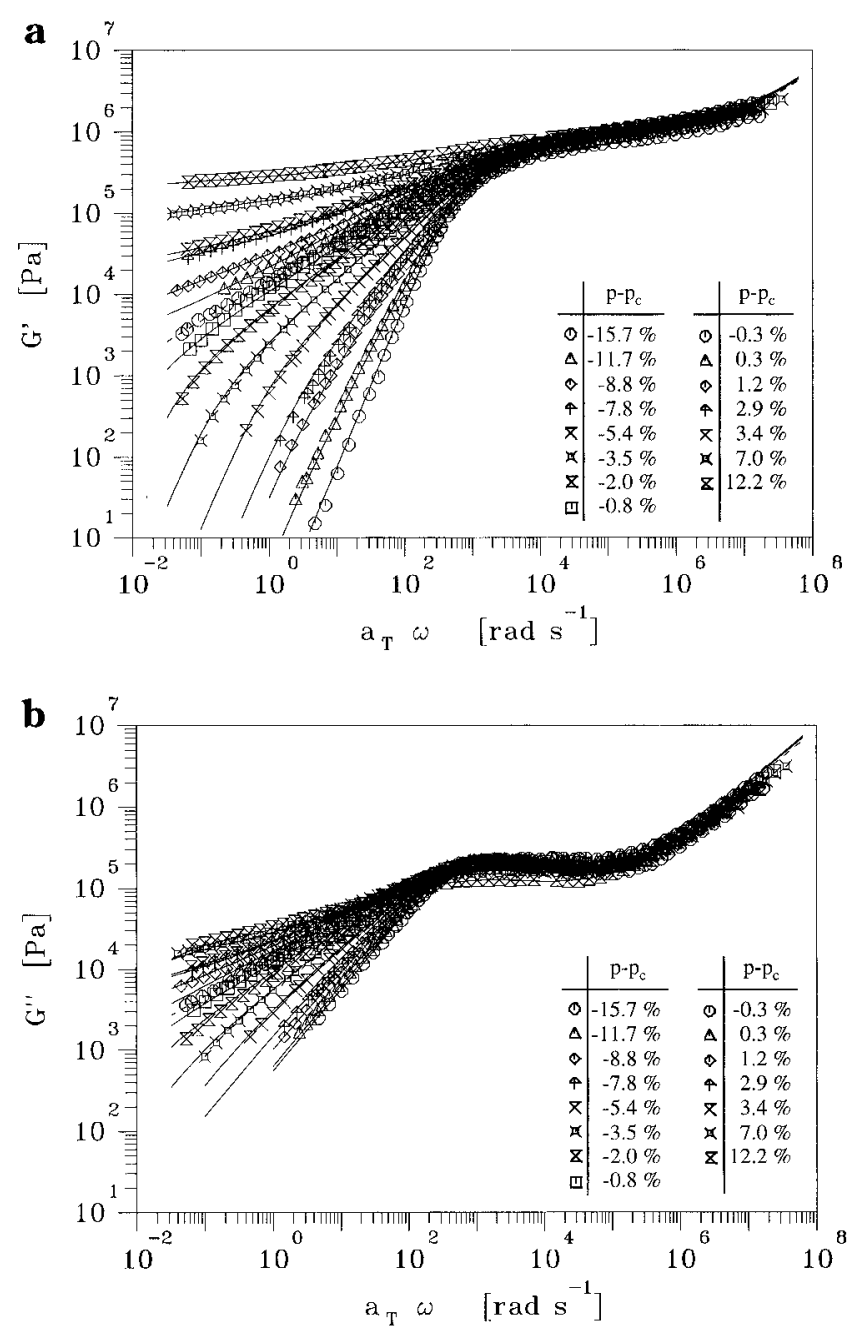

Figure 5. Dynamic moduli data (symbols) and model (line) of stopped PBD38 samples at different extents of reaction ( $T_{\text {ref }}$ $=28^{\circ} \mathrm{C}$ ): (a) storage modulus, G'; (b) loss modulus, $\mathrm{G}^{\prime \prime}$. The gel point is marked by a dashed line.

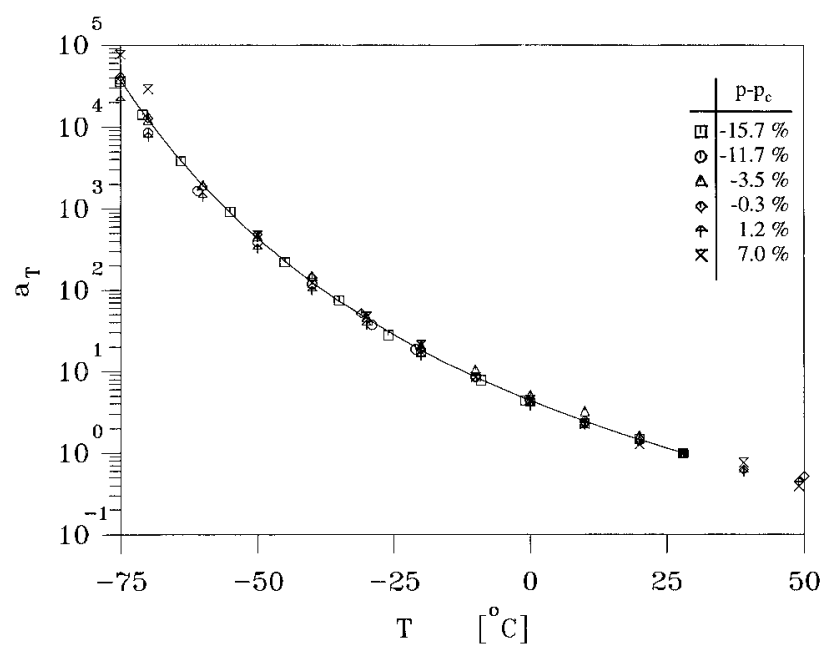

Figure 6. Horizontal shift factors, $\mathrm{a}_{\mathrm{T}}$, for several PBD38 samples before and after the gel point. The line represents the WLF fit for the uncross-linked sample.

with interpolated "snapshot" data. The accuracy in determining the best time, $t_{i}$, for each sample lies within $\pm 20 \mathrm{~s}$. For each time, $t_{i}$, during the CFS experiment, the concurrently performed FTIR measurement yielded a corresponding extent of reaction $p\left(t_{i}\right)$. Knowing the reaction times that correspond to the stopped samples from the described superposition of stopped sample

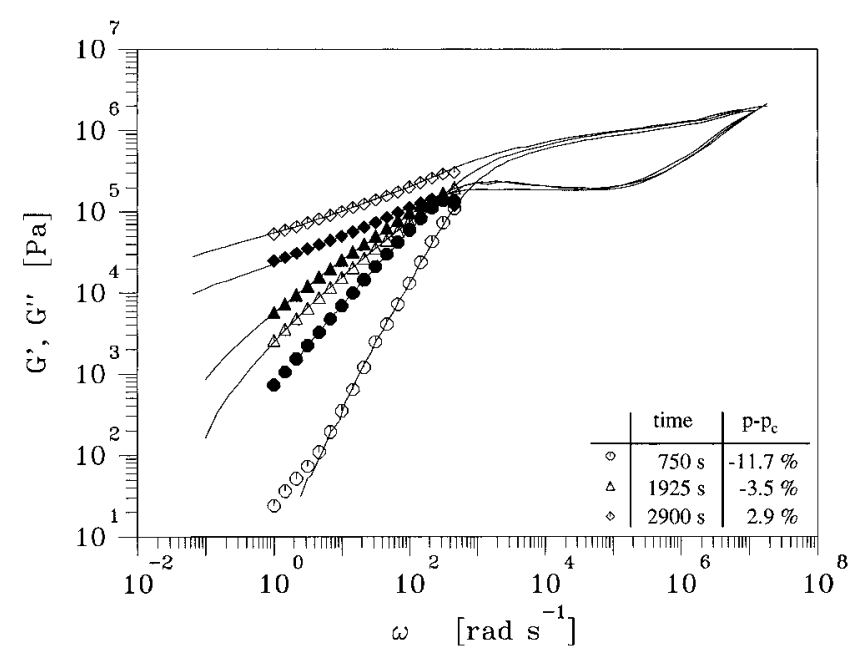

Figure 7. Superposition of stopped sample master curves and interpolated data from CFS experiment for PBD38.

master curves and interpolated dynamic moduli, the FTIR experiment now yields the respective extent of reaction. Although the catalyst poison takes some time to diffuse through the entire sample thickness and the sample therefore is not completely homogeneous, the superposition between stopped sample data (an average of the different cross-linking stages in the sample) and interpolated CFS data (at a single cross-linking stage) is very good. Therefore, the relaxation behavior of the slightly inhomogeneous stopped sample is characteristic for a material with a single extent of reaction, $\mathrm{p}\left(\mathrm{t}_{\mathrm{i}}\right)$. The extents of reaction of all stopped samples of PBD18 and PBD38 as determined by this method are given in Figures 4 and 5 , respectively.

\section{Relaxation Patterns and Model Spectrum}

We propose to use modeling as a tool for analyzing the experimental observations and for detecting characteristic patterns in the behavior. We attempt to cast all the rheological observations near the gel point into a model for the relaxation time spectrum. As an objective, we look for the simplest model because it reveals patterns in the relaxation behavior and intercorrelations between the different observations.

The modeling of the $\mathrm{G}^{\prime}, \mathrm{G}^{\prime \prime}$ data consists of four major steps: (1) calculation of the relaxation spectra of networks at different extents of reactions (using the method of Baumgärtel and Winter ${ }^{28}$ ), (2) determination of characteristic relaxation patterns, (3) abstraction into a simple model and evaluation of model parameters, and (4) application of this model to predict the transient relaxation behavior during the gelation process and certain other rheological observations at the gel point, thus checking the model's self-consistency. The result should be, if the modeling is successful, a unified and self-consistent picture of the gelation process.

Precursor Spectrum. The relaxation behavior of the nearly monodisperse polymer precursor follows a distinct pattern which can be described by the BSW relaxation time spectrum. ${ }^{31,32}$ The spectrum consists of a superposition of two power laws with slopes $-n_{g}$ and $n_{e}$ describing the glass transition and entangl ement regime, respectively:

$$
\begin{gathered}
H(\lambda)=n_{e} G_{N}^{\circ}\left[\left(\lambda / \lambda_{c}\right)^{-n_{g}}+\left(\lambda / \lambda_{e}\right)^{n_{e}}\right] \\
\text { for } \lambda<\lambda_{e}
\end{gathered}
$$


$\lambda_{c}, \lambda_{e}$, and $G_{N}{ }^{\circ}$ denote a characteristic relaxation time for the glass transition, the longest relaxation time, and the plateau modulus, respectively.

The BSW spectrum is the simplest spectrum known to be able to describe G', G" data of polymers with linear flexible molecules of uniform molecular weight. More complicated proposals can be found in the literature, but their increased level of detail is not sufficiently supported by data. A more detailed spectrum cannot be extracted from the data due to the Morozov discrepancy principle. ${ }^{33}$ The Morozov principle expresses the fact that a physical model for describing a data set cannot be of higher accuracy than the noise in the data. For this reason, we decided to stay with the simplest choice of sufficient accuracy, the BSW spectrum.

It is important to note that the addition of the crosslinking agent plasticizes the material, but the mixture can still be described by a BSW spectrum. However, BSW parameters for the pure polymer and the mixture of polymer and cross-linker differ, as determined by De Rosa and Winter. ${ }^{4}$

Model Extension for Nearly Critical Gels. (i) Before the Gel Point. The cross-linking reaction permanently connects precursor molecules and thereby introduces longer relaxation times which have to be taken into account. This is accomplished by adding a third power law with slope $-\mathrm{n}$ (CW spectrum) to the precursor spectrum. Beyond the longest relaxation time of the precursor (cutoff of the original BSW spectrum), $\lambda_{\mathrm{e}}$, the CW spectrum represents the relaxation behavior in the terminal zone up to the growing longest relaxation time, $\lambda_{\max }\left(\lambda_{\mathrm{e}}<\lambda<\lambda_{\max }\right)$. This longest relaxation time increases with growing cluster size (increasing extent of reaction) and, at the gel point, diverges to infinity. The relaxation exponent $\mathrm{n}$ can also be a function of $p .19,20$ When modeling the relaxation spectrum of critical gels of high molecular weight model polybutadiene precursors with a combination of BSW and CW spectra, De Rosa and Winter ${ }^{4}$ found it necessary to decrease the slope of the entanglement power law, $\mathrm{n}_{\mathrm{e}}$, which is a possibility also considered in the present work by introducing a modified slope, $A n_{e}$, in this region.

(ii) Beyond the Gel Point. The experimental data of materials after the gel point required the addition of a boxlike contribution to the spectrum (termed $\mathrm{H}_{\infty}$ ) in order to be modeled satisfactorily. A simple decrease of the relaxation exponent $n$, for example, could not model the increase in $G^{\prime \prime}(p)$ after the gel point. Furthermore, the equilibrium modulus, $\mathrm{G}_{\mathrm{e}}$, is finite beyond the gel point, characterizing the elastic behavior of the material at low frequencies.

Based on these results, the following spectrum is proposed:

$$
\begin{aligned}
& H(\lambda)= \\
& \begin{cases}n_{e} G_{N}{ }^{o}\left(\lambda / \lambda_{c}\right)^{-n_{g}}+H\left(\lambda_{e}\right)\left(\lambda / \lambda_{e}\right)^{A n_{e}} & \text { for } 0<\lambda<\lambda_{e} \\
\left(H\left(\lambda_{e}\right)-H_{\infty}\right)\left(\lambda / \lambda_{e}\right)^{-n}+H_{\infty} & \text { for } \lambda_{e}<\lambda<\lambda_{\max }\end{cases}
\end{aligned}
$$

The BSW part of the spectrum $\left(0<\lambda<\lambda_{\mathrm{e}}\right)$ describes the entanglement and glass transition behavior, and the CW part $\left(\lambda_{\mathrm{e}}<\lambda<\lambda_{\max }\right)$ represents the terminal zone relaxation. The parameter $\mathrm{H}_{\infty}$ is zero before the gel point and carries a positive value after GP. Figure 8 shows a schematic of this spectrum before and after the gel point. This simple model is capable of describing the inherent relaxation patterns of nearly critical gels.

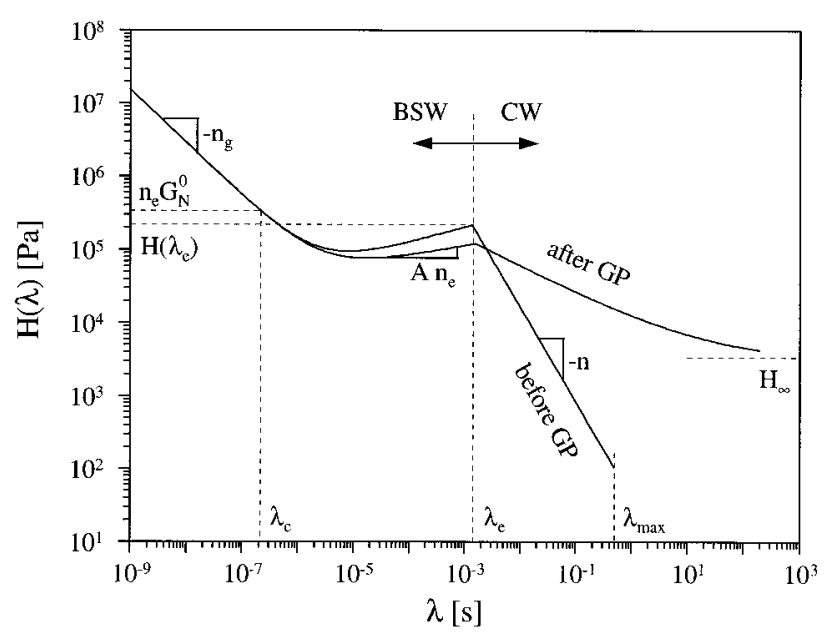

Figure 8. Schematic of the model relaxation spectra before and after the gel point.

The height of the entanglement power law is lowered from the precursor value of $n_{e} G_{N}{ }^{\circ}$ to $H\left(\lambda_{e}\right)$, which can be calculated as follows without introducing any additional parameter: the plateau modulus $\mathrm{G}_{\mathrm{N}}{ }^{\circ}$ (which can increase with increasing conversion) is related to the loss modulus without the glassy contribution $\mathrm{G}_{\text {ent.,term. }}{ }^{34}$

$$
\mathrm{G}_{\mathrm{N}}^{\circ}=\frac{2}{\pi} \int_{-\infty}^{\infty} \mathrm{G}_{\text {ent.,term. }}^{\prime \prime}(\omega) \mathrm{d} \ln \omega
$$

which can be converted into

$$
\mathrm{G}_{\mathrm{N}}{ }^{\circ}=\int_{0}^{\lambda_{\max }} \mathrm{H}_{\text {ent.,term. }}(\lambda) \frac{\mathrm{d} \lambda}{\lambda}
$$

The integration in eq 14 results in

$$
H\left(\lambda_{\mathrm{e}}\right)=\frac{\mathrm{G}_{\mathrm{N}}{ }^{\circ}+\left(\mathrm{H}_{\infty} / \mathrm{n}\right)\left[1-\left(\lambda_{\max } / \lambda_{\mathrm{e}}\right)^{-\mathrm{n}}-\mathrm{n} \ln \left(\lambda_{\text {max }} / \lambda_{\mathrm{e}}\right)\right]}{\frac{1}{\mathrm{An} n_{\mathrm{e}}}+(1 / \mathrm{n})\left[1-\left(\lambda_{\text {max }} / \lambda_{\mathrm{e}}\right)^{-\mathrm{n}}\right]}
$$

The parameter set can therefore be split into two parts, one set describing the precursor relaxation $\left(\mathrm{G}_{\mathrm{N}}{ }^{\circ}, \mathrm{n}_{\mathrm{e}}, \mathrm{n}_{\mathrm{g}}\right.$, $\left.\lambda_{c}, \lambda_{\mathrm{e}}\right)$ and the second set describing the modifications of this relaxation behavior due to gelation $\left(A, n, \lambda_{\max }\right.$ before the gel point; $A, n, \lambda_{\max }, H_{\infty}, G_{e}$ after the gel point). The precursor parameter set is evaluated for the first sample of both materials (no cross-linking) and remains unchanged for all other samples. The remaining parameters are evaluated using IRIS DS (Iris Devel opment System) software. The evaluation procedure is described in more detail in the supporting information available for this paper. Figures 4 and 5 show the comparison between experimental and calculated dynamic moduli for the stopped samples of PBD18 and PBD38, respectively.

The relaxation exponent $n$ decreased with increasing extent of reaction, as shown in Figure 9 for PBD18 and PBD38. The inset in this figure depicts the behavior of parameter A with extent of reaction for the PBD18 example.

In addition to the longest relaxation time, $\lambda_{\max }$, before and after the gel point, eqs 7 and 8 , and to the equilibrium modulus, $\mathrm{G}_{\mathrm{e}}$, eq 6 , the parameter $\mathrm{H}_{\infty}$ also follows a characteristic scaling law:

$$
\mathrm{H}_{\infty} \propto\left(\mathrm{p}-\mathrm{p}_{\mathrm{c}}\right)^{\beta}
$$




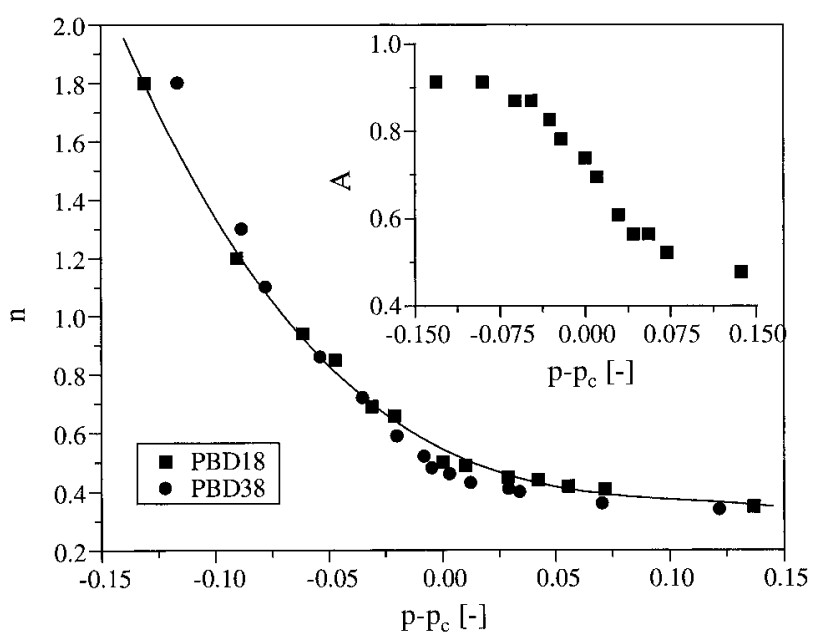

Figure 9. Evolution of relaxation exponent $\mathrm{n}$ with extent of reaction for PBD18 and PBD38. The line represents a third order polynomial approximation of the PBD18 data. The inset depicts the dependence of parameter $A$ (decrease of entanglement exponent $\mathrm{n}_{\mathrm{e}}$ ) for PBD18.

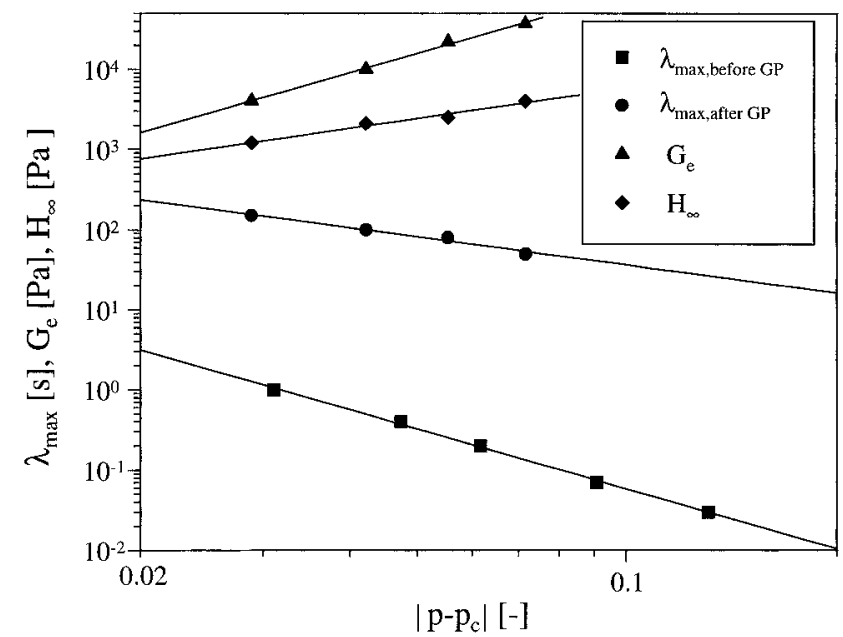

Figure 10. Scaling of parameters $\lambda_{\max }, \mathrm{G}_{\mathrm{e}}$, and $\mathrm{H}_{\infty}$ with distance from the gel point, $\left|p-p_{c}\right|$, for PBD18. Symbols depict the values extracted from modeling the stopped sample master curves, and lines represent the corresponding fit by linear regression.

Table 1. Comparison of Scaling Exponents Predicted by Several Gelation Theories and Experimental Scaling Exponents for Our Model

\begin{tabular}{lrlllll}
\hline & $\mathrm{n}$ & $\mathrm{s}$ & $\mathrm{z}$ & $\alpha_{-}$ & $\alpha_{+}$ & $\beta$ \\
\hline \multicolumn{7}{c}{ Theoretical10 } \\
Flory-Stockmayer & 1 & 0 & 3 & 0 & 3 & \\
network with Rouse dynamics & $2 / 3$ & $4 / 3$ & 2.67 & 4 & 4 & \\
electric network analogy & 0.71 & 0.75 & 1.94 & 2.59 & 2.73 \\
\multicolumn{7}{c}{ Experimental } \\
PBD18 & 0.5 & 1.16 & 2.39 & 2.48 & 1.16 & 1.26 \\
PBD38 & 0.45 & 1.19 & 1.97 & 2.42 & 1.35 & 1.00
\end{tabular}

The scaling of these parameters for PBD18 is given in Figure 10. Scaling exponents which are valid for our model were found by linear regression and are listed in Table 1.

Self-Consistency of the Model. The final modeling step is the self-consistency test. The model spectrum and its dependence on $\mathrm{p}$ were used to calculate the evolution of dynamic moduli with extent of reaction (Figure 11). The functional dependence of $n$ and $A$ on extent of reaction can be very closely approximated by a third order polynomial and a sigmoidal function, respectively, for this purpose (these functions are used

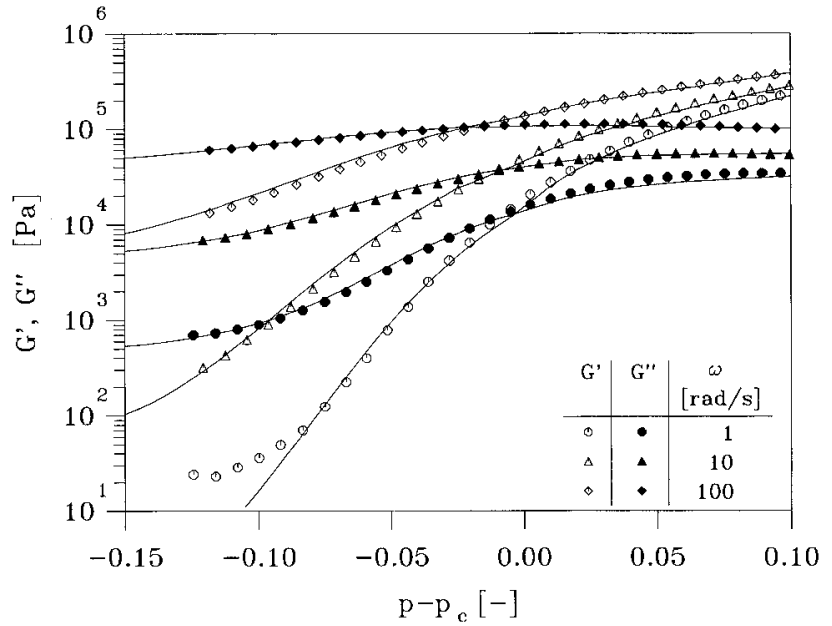

Figure 11. Evolution of dynamic moduli during cross-linking of PBD38 at $28^{\circ} \mathrm{C}$. Comparison of data (symbols) and model (line) at three different frequencies (open symbols, G'; filled symbols, G").

only to simplify the modeling process; no physical significance is given to the functional form). The scaling equations (eqs 6-8 and 16) are used to calculate the model parameter at several extents of reaction. This restricts the model to the vicinity of $p=p_{c}$ since the power laws should break down at some distance from $\mathrm{p}_{\mathrm{c}}$. This distance is not known beforehand and has to be determined in the course of the study.

The model also describes other experimental observations near and at the gel point. The divergence of zeroshear viscosity and equilibrium compliance is predicted, and power law exponents for the divergence of $\eta_{0}$ can be found ( $\mathrm{s}=1.16$ for PBD18 and $\mathrm{s}=1.19$ for PBD38).

Using the proposed model combination of BSW and CW spectra, the gel stiffness is found (using eqs 2 and 12 and some simplifications) to be

$$
\mathrm{S} \cong \mathrm{n}_{\mathrm{e}} \Gamma(\mathrm{n}) \mathrm{G}_{\mathrm{N}}{ }^{0} \lambda_{\mathrm{e}}{ }^{\mathrm{n}}
$$

This is of the same basic form as the experimentally observed relation between the gel stiffness, $\mathrm{S}$, and the relaxation exponent, $\mathrm{n}: 35,36$

$$
\mathrm{S}=\mathrm{G}_{0} \lambda_{0}{ }^{\mathrm{n}}
$$

with the crossover relaxation time, $\lambda_{0}$, equal to the longest relaxation time of the polymer precursor, $\lambda_{e}$, and $\mathrm{G}_{0}$ equal to $n_{\mathrm{e}} \Gamma(n) \mathrm{G}_{N}{ }^{\circ}$. Since $n_{\mathrm{e}} \Gamma(n)$ is in the order of unity, $G_{0}$ is found to be in the order of magnitude of the plateau modulus, $\mathrm{G}_{\mathrm{N}}{ }^{\circ}$. Likewise, $\mathrm{G}_{0} \lambda_{0}$ can be calculated to be approximately the magnitude of the zero-shear viscosity of the precursor, $\eta_{0}$. This agrees with the experimental observations. ${ }^{35,36}$

Another experimental observation, 4,23 the scaling of the rates of change of the dynamic moduli at the gel point with frequency (in the terminal zone),

$$
\frac{\partial \ln \mathrm{G}^{\prime}}{\partial \mathrm{p}} \propto \frac{\partial \ln \mathrm{G}^{\prime \prime}}{\partial \mathrm{p}} \propto \omega^{-\kappa}
$$

(with a value for the exponent $\kappa$ of approximately 0.2 ), is also described by our model.

A more in-depth discussion of these observations and the corresponding model predictions is given in the supporting information.

\section{Discussion}

Our rheological experiments on nearly critical gels from high molecular weight precursors exhibit a char- 
acteristic relaxation pattern that can be described with our phenomenological model. The new spectrum, eq 12, has a very simple functional form since it only consists of a superposition of several power laws. It therefore represents an extension of successful power law relaxation time spectra as applied to nearly monodisperse linear flexible polymers, ${ }^{31}$ bimodal blends of such polymers, ${ }^{37}$ and branched polymers with uniform arm lengths. ${ }^{38}$ Besides this inherent simplicity, the parameters of our new model show interesting p-dependence. The relaxation exponent $\mathrm{n}$ is not constant but changes with $p$, and the plateau modulus $G_{N}{ }^{0}$ increases slightly with increasing $p$ due to the addition of cross-links in the material. The entanglement slope of the precursor spectrum, $n_{e}$, (expressed by the parameter A) changes as well, which can be understood in terms of polydispersity effects. Polydispersity due to random crosslinking introduces a broadening of the spectrum. ${ }^{39}$

Furthermore, a new contribution to the spectrum, $\mathrm{H}_{\infty}$, appears after the gel point for reasons which are not completely understood yet. It adds a nearly constant value to $G^{\prime \prime}$ for frequencies above $\omega \approx 1 / \lambda_{\text {max }}$. Below this frequency, the drop-off to liquid-like behavior $\left(G^{\prime \prime} \propto \omega^{2}\right)$ can be seen. $H_{\infty}$ also affects $G^{\prime}$ as well as the relaxation modulus $G(t)$, however, less significantly since, after the gel point, the equilibrium modulus $\mathrm{G}_{\mathrm{e}}$ dominates the behavior of $\mathrm{G}^{\prime}$ at low frequencies and of $\mathrm{G}(\mathrm{t})$ at long times. Ferry ${ }^{34}$ noticed a similar trend in the relaxation spectrum of cross-linked amorphous polymers. He attributed this to some continuing degree of relaxation (although low) at long times. This contribution of $\mathrm{H}_{\infty}$ strongly resembles the box of Tobolsky's wedge-box spectrum, ${ }^{40}$ which he used in an attempt to describe the entanglement relaxation. This suggests that the contribution of $\mathrm{H}_{\infty}$ might arise due to an additional longrange constraint imposed on the relaxation process. A possible cause might be large molecular clusters that are trapped by the infinite network. If, with increasing extent of reaction, additional clusters develop, then they could lead to an increase in $\mathrm{H}_{\infty}$ and $\mathrm{G}^{\prime \prime}$. On the other hand, more and more clusters connect to the network and, thus, contribute no further to the loss modulus. After a sufficient degree of cross-linking, more clusters connect to the network than are being formed. This results in a maximum of $\mathrm{G}^{\prime \prime}$ and $\mathrm{H}_{\infty}$ and a subsequent decrease at a certain distance beyond the gel point. A physical model that would directly predict such relaxation behavior could not be found in the literature.

The contribution of $\mathrm{H}_{\infty}$ has to be distinguished from the equilibrium modulus, $\mathrm{G}_{\mathrm{e}}$, which al so has a non-zero value only after the GP. The equilibrium modulus only contributes to $\mathrm{G}^{\prime}$ and $\mathrm{G}(\mathrm{t})$, and it adds a constant to both material functions for all frequencies and times, respectively. The box $\mathrm{H}_{\infty}$ is part of the spectrum. Its contribution appears in $\mathrm{G}^{\prime}$ and $\mathrm{G}^{\prime \prime}$, as well as in $\mathrm{G}(\mathrm{t})$. However, it does not contribute below $\omega \approx 1 / \lambda_{\max }$ (for $\mathrm{G}^{\prime}$ and $\mathrm{G}^{\prime \prime}$ ) and beyond $\mathrm{t} \approx \lambda_{\max }$ (for $\left.\mathrm{G}(\mathrm{t})\right)$. The difference can be seen when using the following equation to calculate the relaxation modulus from the spectrum: ${ }^{34}$

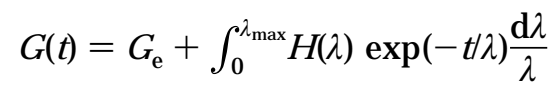

$\mathrm{G}_{\mathrm{e}}$ is time independent and contributes to $\mathrm{G}(\mathrm{t})$ at all times. The contribution of $\mathrm{H}(\lambda)$ (and therefore al so $\mathrm{H}_{\infty}$ ) to the relaxation modulus is, however, time dependent because of the exponential in the integral.

Sensitivity Analysis. The model is quite sensitive to small variations of the parameters, which allows determination of the parameters within narrow margins. Before the gel point, the parameters $\left(A, n, \lambda_{\max }\right)$ can be evaluated nearly independently of each other. This results in errors of approximately $\pm 5 \%$. After the gel point, $A$ and $n$ can still be considered independent, but $\lambda_{\text {max }}, G_{e}$, and $H_{\infty}$ have to be evaluated simultaneously and affect the same region of the relaxation response. Furthermore, the accuracy of the data determination suffers if the low-frequency range of the sample is not fully covered by the experimental window. This is the case with the PBD38 samples beyond GP. Only the lower limit of the longest relaxation time could be evaluated. Since the choice of $\lambda_{\max }$ influences the height of the storage modulus at low frequencies, it also affects the suitable choi ce for the equilibrium modulus. For these reasons, the error involved with parameter evaluation is more pronounced after the gel point (around $\pm 15 \%$ ) than it is before the gel point.

Matching of the Data Sets. Two types of data sets were used in the parameter evaluation; both came from the same sample batch but differ in the experimental procedure. The first data set is measured on stopped samples over a wide range of frequencies, but without knowing the specific extent of reaction. The second data set is taken during the cross-linking process, giving $\mathrm{G}^{\prime}$, $G^{\prime \prime}(\omega, t)$ data from the rheometer and $p(t)$ data from FTIR. The disadvantage of the second data set is its very small frequency window. By matching the two data sets visually on the computer screen, it is possible to assign specific $\mathrm{p}(\mathrm{t})$ values to the stopped samples. These values are used to describe the $p$-dependence of the rheological parameters in the gelation model.

Critical Extent of Reaction and Parameter Scaling. Errors in the estimation of times $t_{i}$ for the stopped samples and the critical gel time, $t_{c}$, from the CFS experiment and possible discrepancies between the temperatures in the rheometer and the infrared spectrometer lead to small errors of about \pm 0.006 in the determination of $p$ and $p_{c}$. Also, the relative error in $\mid p$ - $p_{c}$ (important in deriving the scaling behavior) is small since $\left|p-p_{c}\right|$ is greater than $1 \%$ for the samples considered in the evaluation of the $p$-dependence of the model parameters.

Subsequently, the model parameter dependence on extent of reaction (or distance from the gel point, $\mid p-$ $\left.p_{c}\right)$ could be investigated. The parameters $\lambda_{\text {max }}, G_{e}$, and $\mathrm{H}_{\infty}$ follow characteristic scaling relations with the distance from the gel point. Table 1 gives a comparison of experimental scaling exponents and scaling exponents from several gelation theories. It can be clearly seen that neither of the theories predicts the exponents found in this study. Therefore, the parameters of our model spectrum cannot be predicted from prevailing gelation theories. This is probably due to the fact that percolation theory does not apply to our system except very close to the gel point. ${ }^{14}$ Also, our approach to those parameters and scaling exponents differs from the approach taken in the theories, and the values cannot necessarily be compared directly.

The assumption that $\lambda_{\max }$ exhibits symmetric scaling before and after the gel point ${ }^{15}$ could not be verified for our model; in fact, the exponents $\alpha_{-}$and $\alpha_{+}$differ significantly. The scaling exponents are very sensitive to small changes in $\left|p-p_{c}\right|$ as recently confirmed by Lusignan et al.18 Our method of determining the gel point from the $\tan \delta$ crossover and the distance from the gel point by matching our data sets is very precise. Calculations of the largest possible error when using 
combinations of the previously given error bounds for $\left|p-p_{c}\right|$ and $\lambda_{\max }$ result in absolute errors of approximately \pm 0.15 for $\alpha_{-}$and $\alpha_{+}$. The conclusion of nonsymmetric scaling of $\lambda_{\max }$ is not affected, even when using these largest possible errors.

Relaxation Exponent $\mathbf{n}$. A constant value for the relaxation exponent, $n$, in the vicinity of the gel point has been hypothesized repeatedly in the literature. ${ }^{22,24,41}$ A constant $n$ value would allow time-cure superposition, which was first proposed by Adolf and Martin. ${ }^{22}$ Hodgson and Amis ${ }^{41}$ confirmed this principle on a slightly different system. Both studied gelation in epoxy systems, starting from monomer precursors.

A constant $n$ value is also required for the validity of dynamic scaling. ${ }^{8,9}$ Rubinstein et al. ${ }^{21}$ applied this dynamic scaling theory to dynamic moduli data of several stopped samples from condensation of diesters with alcohol. The theory, with its prediction of a constant $n$ value, does not result in particularly good agreement between model and experimental data.

In this study, it was found that $\mathrm{n}$ is a function of extent of reaction for the investigated cross-linking system (starting from high molecular weight precursors). Therefore, time-cure superposition was not applicable here. Horizontal and vertical shifting of the termi nal zone data did not yield a unique master curve. The time-cure superposition might work for small molecules but not for our system of high molecular weight precursor.

\section{Conclusions}

The model polymer combines the relaxation characteristics of a nearly monodisperse linear precursor material with the broadening of the relaxation spectrum due to cross-linking. The gelation process introduces broad polydispersity (at GP, the polydispersity index diverges); however, the characteristic relaxation features of the monodisperse precursor are still distinguishable at states significantly beyond the gel point. Major changes to the spectrum are seen in the terminal zone, where the increasing network connectivity results in the appearance of Ionger relaxation modes. These slow modes are distributed as a power law with the exponent $\mathrm{n}$ decreasing with increasing extent of reaction. At the same time, the longest relaxation time increases before and decreases again after the gel point. The entanglement and glass transition regime are dominated by the precursor's relaxation behavior, except for a decrease in the entangl ement power law exponent due to polydispersity effects.

By observing characteristic patterns in the relaxation of samples for which the cross-linking reaction was stopped near the gel point, we were able to formulate a phenomenological model for the relaxation time spectrum in the vicinity of the gel point using only a small number of meaningful parameters. An appropriate choice of these parameters resulted in good agreement between model and experimental data on a large number of stopped samples over a wide frequency window. Despite its simplicity (superposition of several power laws), the new model is able to describe all rheol ogical observations in the vicinity of the gel point.

Several basic questions could be answered experimentally. The most important concerns the relaxation spectrum at long times. It may be represented by a power law (self-similar relaxation) of slope $\mathrm{n}$ which is cut off at the longest relaxation time. However, we found the value of $n$ to decrease significantly with increasing extent of reaction. This implies that dynamic scaling theory and time-cure superposition do not apply to our system of cross-linked polymers from high molecular weight precursors, at least in the investigated range of distances from the gel point.

The longest relaxation time, $\lambda_{\max }$, and the equilibrium modulus, $G_{e}$, scale with distance from the gel point, Ip $-p_{c}$. The scal ing exponents for those parameters are valid for our phenomenological model, but they cannot be predicted by any of the prevailing gelation theories since a different approach to those parameters is used. Interestingly, the scaling exponents for the longest relaxation time before and after the gel point differ significantly with a stronger dependence before the gel point.

The relaxation pattern of polymers near the gel point was analyzed in terms of a few parameters and a simple model approximation. The physical meaning of these parameters will have to be explored further. The observed patterns are intended to give guidance to theoretical approaches to gelation such as molecular dynamics and percolation.

Acknowledgment. We gratefully acknowledge financial support from the $\mathrm{National} \mathrm{Science} \mathrm{Foundation}$ through the Materials Research and Engineering Center at the University of Massachusetts and from Raychem Corp. M.M. is indebted to the German Academic Exchange Service (DAAD-Doktorandenstipendium aus Mitteln des zweiten Hochschul sonderprogramms) for financial support. We thank Prof. S. L. Hsu for giving us access to his FTIR facilities and Dr. M. Masse and Dr. J. Machado for providing the polybutadiene standards.

Supporting Information Available: Description of the procedure for evaluating model parameters and an in-depth discussion of the experimental observations and corresponding model predictions (12 pages). See any current masthead page for ordering information.

\section{References and Notes}

(1) Chambon, F.; Winter, H. H. Polym. Bull. 1985, 13, 499.

(2) Winter, H. H.; Chambon, F. J . Rheol. 1986, 30, 367.

(3) Chambon, F.; Winter, H. H. J . Rheol. 1987, 31, 683.

(4) De Rosa, M. E.; Winter, H. H. Rheol. Acta 1994, 33, 220.

(5) Stauffer, D.; Coniglio, A.; Adam, A. Adv. Polym. Sci. 1982, $44,103$.

(6) Stauffer, D. Introduction to Percolation Theory; Taylor and Francis Inc.: Philadel phia, PA, 1985.

(7) de Gennes, P. G. Scaling Concepts in Polymer Physics; Cornell University Press: I thaca, NY, 1979.

(8) Daoud, M. J. Phys. A 1988, 21, L237.

(9) Martin, J . E.; Adolf, D.; Wilcoxon, J . P. Phys. Rev. Lett. 1988, 61,2620

(10) Martin, J . E.; Adolf, D. Annu. Rev. Phys. Chem. 1991, 42, 311.

(11) de Gennes, P. G. J . Phys. Lett. 1976, 37, 1

(12) Flory, P. J. J . Am. Chem. Soc. 1941, 63, 3083.

(13) Stockmayer, W. H. J . Chem. Phys. 1943, 11, 45.

(14) de Gennes, P. G. J . Phys. Lett. 1977, 38, 355.

(15) Winter, H. H. Progr. Colloid Polym. Sci. 1987, 75, 104.

(16) Durand, D.; Delsanti, M.; Adam, M.; Luck, J . M. Europhys. Lett. 1987, 3, 297.

(17) Goldenfeld, N.; Goldbart, P. Phys. Rev. A 1992, 45, R5343.

(18) Lusignan, C. P.; Mourey, T. H.; Wilson, J. C.; Colby, R. H. Phys. Rev. E 1995, 52, 6271.

(19) Friedrich, C.; Heymann, L. J . Rheol. 1988, 32, 235.

(20) Friedrich, C.; Heymann, L.; Berger, H. R. Rheol. Acta 1989, $28,535$.

(21) Rubinstein, M.; Colby, R. H.; Gillmor, J . R. Springer Ser. Chem. Phys. 1989, 51, 66.

(22) Adolf, D.; Martin, J. E. Macromolecules 1990, 23, 3700.

(23) Scanlan, J. C.; Winter, H. H. Makromol. Chem., Macromol. Symp. 1991, 45, 11.

(24) Winter, H. H. MRS Bull. 1991, 16, 44. 
(25) Mours, M.; Winter, H. H. Rheol. Acta 1994, 33, 385.

(26) Vallés, E. M.; Macosko, C. W. Macromolecules 1979, 12, 521.

(27) Winter, H. H.; Baumgärtel, M.; Soskey, P. R. In Techniques in Rheological Measurement; Collyer, A. A., Ed.; Chapman $\&$ Hall: London, 1993.

(28) Baumgärtel, M.; Winter, H. H. Rheol. Acta 1989, 28, 511.

(29) Gruver, J . T.; Kraus, G. J . Polym. Sci. Part A 1964, 2, 797.

(30) Kasehagen, L. J .; Macosko, C. W.; Trowbridge, D.; Magnus, F. J . Rheol. 1996, 40, 689 .

(31) Baumgärtel, M.; Schausberger, A.; Winter, H. H. Rheol . Acta 1990, 29, 400.

(32) Baumgärtel, M.; De Rosa, M. E.; Winter, H. H.; Machado, J .; Masse, M. Rheol. Acta 1992, 31, 75.

(33) Morozov, V. Methods for Solving I ncorrectly Posed Problems; Springer: Berlin, 1984.
(34) Ferry, J . D. Viscoelastic Properties of Polymers; Wiley and Sons Inc: New York, 1980.

(35) Scanlan, J. C.; Winter, H. H. Macromolecules 1991, $24,47$.

(36) I zuka, A.; Winter, H. H.; Hashimoto, T. Macromol ecules 1992, $25,2422$.

(37) J ackson, J . K.; Winter, H. H. Macromolecules 1995, 28, 3146.

(38) J ackson, J . K. Ph.D. Dissertation, University of Massachusetts, Amherst, MA, 1995.

(39) Schosseler, S.; Leibler, L. J . Phys. Lett. 1984, 45, 501.

(40) Tobolsky, A. V. Properties and Structure of Polymers; Wiley \& Sons Inc: New York, 1960.

(41) Hodgson, D. F.; Amis, E. J . Macromolecules 1990, 23, 2512. MA9517097 